\title{
EDITORIAL
}

\section{THE NEONATAL EYE}

The ability to keep infants of low gestational age alive has made all ophthalmologists acutely aware of the problems of the eye in the neonate and the difficulties involved in examining and correctly diagnosing disease from the side of the cot, whilst being entangled in masses of wires and tubes. Nor are they helped by the fact that the eye is minute or disappears behind tightly closed eyelids.

The defects that arise, or which are detected during the neonatal period, are usually the result of either genetic defects or arrest or delay in the development of the fetus or newborn infant. The mapping of the genes and the ability to remove those embryos affected by inherited disorders will no doubt in the future allow us to eliminate many sources of inherited blindness, so there is now considerable urgency to expand on our knowledge of these relatively common problems.

Exposure to teratogenic agents, such as rubella for cataract, Thalidomide, and, the worst of all, alcohol, leads to irreparable damage to the embryo if it occurs at a critical stage in its development. Studies of infants born during the Thalidomide tragedy have given important clues to the exact timing of the important ocular events in development. For instance, exposure to a pulse of Thalidomide at 20 days after conception results in anotia and incommitant strabismus; 24 days after conception the upper limb defects appear and at 27 days both upper and lower limb defects occur. In between these, many develop Duane's syndrome and/or lacrimal abnormalities, probably because of damage to the VIth nerve, lacrimal, and other brain-stem nuclei. Similarly, defects such as Peter's, Reiger's and Axenfeld's syndromes can be timed to the migration of the three waves of neural crest cells, showing that these defects are not due to anterior chamber cleavage defects as had been previously suspected.

The absolute precision and integration of the steps of development continue after birth as the visual system matures. The 'where' and 'what' control mechanisms which enable us to locate our position in the world and to identify the object of our gaze develop separately, but in parallel, starting from the parvo- and magno-cellular ganglia cells within the retina. Thus, while the newborn infant's visual system is largely sub-cortical, development occurs rapidly during the next six months at a time when there is an extraordinary development of cerebral connectivity. What is remarkable is that each aspect of vision matures at a particular time, thus orientation is at a different time from directional motion and again at a different time of development from visual acuity to stereopsis.

The programming of development, both pre and post-natal, is exact and only amenable to very minor modification. This is not limited to the neonatal period but continues throughout development so that if a phase is missed, it is never learned. Educationalists please note! 